UC-15

Issued: November 1985

LA--10596-MS

DE $86 \quad 005836$

\title{
Confirming LEU in an LFUA Environment
}

\author{
R. R. Picard
}

\section{DISCLAIMER}

\begin{abstract}
This report was prepared as an account of work sponsored by an agency of the United States Government. Neither the United States Government nor any agency thereof, nor any of their employees, makes any warranty, express or implied, or assumes any legal liability or responsibility for the accuracy, completeness, or usefulness of any information, apparatus, product, cr process disclosed, or represents that its use would not infringe privately owned rights. Reference herein to any specific commercial product, process, or service by trade name, trademark, manufacturer, or otherwise does not necessarily constituie or imply its endorsement, recommendation, or favoring by the United States Government or any agency thereof. The views and opinions of authors expressed herein do not necessarily state or reflect those of the United States Government or any agency thereof.
\end{abstract}

\section{MASTER}


R. R. Picard

\section{ABSTRACT}

A standard sequential procedure is adapted to a limited-Erequency unannourced access (LFUA) inspection problem, where uranium enrichment is monitored using nondestructive assay instrumentation. If the enrichment is in the low-enriched uranium (LEU) range, rapid confirmation to that effect is provided under anticipated measurement conditions. Decision thresholds are derived based on the required confidence level in an LEU confirmation. Also, the procedure is easily automated and is such that the raw data need not be revealed.

\section{INTRODUCTION}

A measurement technique to be performed in a limited-frequency unannounced access (LFUA) inspection regime for centrifuge enrichment plants has recently been developed. ${ }^{l}$ This technique, based on an integration of passive gamma-ray spectrometry and $x$-ray fluorescence, measures the enrichment of the gaseous $\mathrm{UF}_{6}$ in the cascade pipework. In accordance with the conclusions ${ }^{2}$ of the Hexapartite safeguards Project, the enrichment measurements are to provide a quick "go/no go" confirmation that the enrichment is in the low-enriched uranium (LEU) range (that is, below 20\%). This report extends an earlier effort, ${ }^{3}$ advocating a standard decision procedure cf sequential analysis for use in the LFUA monitoring problem. Confirmation of LEU is made with 958 confidence, and preliminary results indicate that fast, accurate "go/no go" decisions are reached under anticipated measurement conditions. In secs. II and III, the decision 
procedure is formally defined and background information concerning its use is given. Application to the LFUA problem is discussed in Sec. IV, and simulation work is presented in Sec. $V$ to quantify performance.

II. BACKGROUND: THE SEQUENTIAL PROBABILITY RATIO TEST

Statistical techniques for the evaluation of data olserved sequentially are in widespread use, particularly in the areas of time series and quality control. Safeguards applications are also increasing (see Ref. 4 and the bibliography contaired therein). For the particular problem of enrichment monitoring, it is useful to review a procedure called the Sequential Probability Ratio Test (or SPRT) and attributed to wald. ${ }^{5}$ A modified version of the SPRT is advocated in Sec. $V$ of this report for LFUA work.

The generic decision problem of sequential analysis as related to LFUA monitoring is as follows. Individual enrichment measurements, corresponding to 30-minute counts, are made sequentially. The $i^{\text {th }}$ measured value $m(i)$ is assumed to conform to the model

$$
m(i)=\theta+e(i) \text {, }
$$

where $e(i)$ is the measurement error and $\theta$ denotes the actual enrichment of the $\mathrm{UF}_{6}$ stream. It is presumed that the actual enrichment changes only negligibly during the monitoring perlod. Measurement errors $\{e(i)\}$ are presumed independent and ncrmally distributed with known standard deviation $\sigma$. In practice, of course, such modeling assumptions should be confirmed experimentally; alternative error structures are easily accommodated in the methodology outlined below.

For the moment, suppose that there are only two possible values for the actual enrichment $\theta$, which are denoted by $\theta_{0}$ and $\theta_{1}$ and are known. The problem is to determine which of $\theta_{0}$ and $\theta_{1}$ is in fact the actual value. Moreover, an answer must be obtained quickly and with little chance of reaching an erroneous decision. 
Wald's solution to this generic problem was to sequentially monitor the relative likelihood of $\theta_{0}$ and $\theta_{1}$ and to reach a deciston when that relative likelihood strongly favored one value of $\theta$ over the other. How strongly one value was favored depended on the desired decision error rates. Spectflually, Waid's SPRT proceeds as follows.

(1) Establish the acceptable decision error iates $\alpha$ and $B$. Here, $\alpha$ denotes the acceptable probability that the decision favors $\theta_{1}$ when $\theta_{0}$ is the actual value. Sinilarly, B denotes the acceptable probability that the decision favors $\theta_{0}$ when $\theta_{1}$ is the actual value. Thus, $\alpha$ and $B$ can be thought of as false-alarm rates.

(2) Initialize the procedure by setting $n=1$.

(3) Observe the measured value $m(n)$ and compute the relative likelihood

$$
\left.\lambda(n)=\left\{\prod_{i=1}^{n} \exp -\left[m(i)-\theta_{1}\right]^{2} / 2 \sigma^{2}\right\} / \prod_{i=1}^{n} \exp -\left[m(i)-\theta_{0}\right]^{2} / 2 \sigma^{2}\right\}
$$

(Aside: Were the normal distribution inappropriate for characterizing measurement errors, the relative likelihood $\lambda(n)$ would be computed using a different expression from the above.)

(4) If $\lambda(n)<B /(1-\alpha)$, decide that the actual value is $\theta_{0}$. If $\lambda(n)>(1-B) / \alpha$, decide that the actual value is $\theta_{1}$.

(5) If $B /(1-\alpha) \leq \lambda(n) \leq(1-B) / \alpha$, the relative likelihood is not yet one-sided enough to reach a deciston. Set $n$ to one more than its current value and return to step 3.

The above procedure is very simple to implement in an automated fashion. And, although a comprehensive discussion of the statistical theory is beyond the scope of this report, SPRT has the desirable properties that

- the actual decision error rates can be shown ${ }^{5}$ not to exceed the stated acceptable error rates $\alpha$ and $B$, and 
- decisions are reached very quickly--in Lact, no other test procedure, sequential or otherwise, with the same decision error rates reaches decisions, under either $\theta$ or $\theta_{1}$, with a lower average number of measurements. ${ }^{6-8}$

Because of these desirable properties for the "pure" two-state decision problem, SPRT has berome a very widely used procedure.

\section{THE MODIFIED SPRT}

Despite its low average number of measurements required for decisions, the SPRT can be very slow in isolated cases. The run-length distribution is skewed (in the present application) so that numerous measurements may occasionally be required. Also, if the actual enrichment $\theta$ were between the values $\theta_{0}$ and $\theta_{1}$ specifted by SPRT, the procedure could have a hard time deciding between the two. Indecd, the relatively poor properties of SPRT at certain intermediate values have prompted work 9.10 in developing alternative procedures.

A simple modification to SPRT overcomes the open-endedness of the above five-step iteration. This modification sets a maximum number, $n_{\max }$ ' of measurements that can be obtained. The modifled SPRT proceeds as the unmodified version, and, if no decision has been reached after ( $\left.n_{\max } 1\right)$ measurements, one final measurement is made and a decision is then reached on the basis of the available data.

For the LFUA monitoring problem. the value $n_{\max }=8$ is advocated so that a decision is sure to be forthcoming without undue delay. Because an inspector's time at the facility is limited, prompt decisions are important. Given one half-hour counting per measurement, $n_{\max }=8$ means that a maximum of $A$ hours per decision is involved. The number " 8 " is somewhat arbitrary, as would be any other choice, but it provides a reasonable upper limit to the number of measurements.

other suggested parameter values for the modified SPRT are $\theta=20 \%$ (corresponding to the LEU/HEU threshold), $\theta_{0}=10 \%$, and decision error rates $\alpha=0.1 \%$ and $B=5 \%$. If all eight measurements are required, the 
decisio. to "confirm LEU" is made, provided the relative likelihood $\lambda(8) \leq B /(1-\alpha)$; otherwise, the decision "unable to confirm" is reached. This termination rule guarantees that the decision procedure maintains the error rate $B<5 \%$. In other words, if the actual enrichment is in the highly enriched uranium (HEU) range, then the decision "unable to confirm" is Eorthcoming with $>958$ probability. Thus, following from what logicians call the converse contrapositive, decisions to "confirm LEU" are made with high confidence.

Properties of the advocated procedure are discussed in Sec. $V$, though one point of general interest is made here. It is important to recognize that the acceptable decision error rates $\alpha$ and $B$ established for use in the modified SPRT may or may not be simultaneously achievable. If individual measurements are not of sufficient accuracy, it is not possible to reach high-quality decisions based on eight or fewer such values. In such a case, $\alpha$ is sacrificed in order to maintain $B<5 \%$. The situation is as described in Table $I$. If measured values allow for high confidence in LEU, the "confirm LEU" deciston is reached. If mea ured values allow high confidence in HEU, the "unable to confirm" decisior is reacher Finally, if confidence is not sufficiently high in either LEU or HEU, as could be the case if measurement uncertainties were large, then the "unable to confirm" decision is reached.

TABLE I

DECISIONS UNDEK VARIOUS CONDITIONS

Condition

High confidence in LEU

High confidence in HEU

Confidence is not high
Decision
"Confirm LEU"
"Unable to confirm"
"Unable to confirm" 
IV. THE LFUA MONITORING PROBLEM

The task is to determine whether the actuai enrichment does or does not exceed 20\%. The decision procedure based on observed measurement data must generate a binary result--either a "yes" or a "no"--to that effect; in other words, the ideal detection probability "curve" is a step fun:tion, with the step at $20 \%$ enrichment. Moreover, it is desired that the decision be as timely as possible, be easily automated, and be such that the raw data need not be revealed.

A decision procedure having the ideal detection probability curve is not attainable. Using a modified SPRT, however, leads to decisions that are reached quickly and with relatively little chance of a mistake when

(1) the actual enrichment is in the anticipated range (below 5\%) or

(2) the actual enrichment is in the anomalous range ( $20 \%$ or above). Performance of the modified SPRT depends on the underlying measurement conditions. In the next section, that performance is quantified.

One unavoidable aspect of decisionmaking in the presence of measurement noise is that intermediate enrichments--say, from $12 \%$ to $18 \%$-produce intermediate results. It is simply not possible to construct a reasonable decision procedure for which the detection probability curve is discontinuous. If, Eor example, the probability of the decision "unable to confirm" is equal to $0.1 \%$ when the actual enrichment is $5 \%$ and is equal to 958 when the actual enrichment is $2 n \%$, then the continuity of the detection probability curve implies that there is an intermediate enrichment for which a $50 \%$ detection, say, ensues. In some respects, this is listurbing since certain sub-20\% enrichments generate "unable to confirm" decisions with relatively large probability. The situation is an inevitable consequence, however, of achieving fast and accurate decisions under (1) and (2) above.

problems at intermediate enrichments would tend to disappear if information could become inore perfect so that the detection probability curve would approach the desired step function. In the present case, though, the steepness of the detection probability curve at intermediate enrichments is determined by the quality of the individual measurements (reflected by the standard deviation o) and the quantity of measurements 
(limited to a maximum of eight per decision). Under anticipated ccnditions, the steepness is sufficient co provide exweliciri ciscinination between cases (1) and (2) above; if still better discrimination is desired, it appears that timeliness must be sacrificed.

V. A SUGGESTED PROCEDURE

Consider defining a modified SPRT by establishing the parameter values $\theta_{1}=208, \quad \theta_{0}=10 \%, \quad a=0.18, \quad B=58, \quad$ and $n_{\max }=8$.

The termination rule, as noted in Sec. III, compares $\lambda(8)$ to $B /(1-\alpha)$ in the event that eighi measurements are required. As will be shown, this SPRT gives relatively fast and accurate decisions for anticipated enrichments. More generally, parameter values can be altered to yield a sequential test procedure with somewhat different properties, and the class of modifled SPRT tests is quite useful for LFUA monitoring.

To illustrate, suppose that the standard deviation of each individual measurement is $\sigma=4 \%$. This standard deviation corresponds to good--but under some circumstances, expected--measurement conditions. The modified SPRT defined using the above parameter values is depicted in Fig. 1.

For this procedure, if the first measurement is less than $10.2 \%$, the decision "confirm LEU" is reached immediately. If the first measurement exceeds $26.0 \%$, the decision "unable to confirm" is reached immediately. If the first measurement $\dot{i} \mathrm{al} 1 \mathrm{~s}$ between $10.2 \%$ and $26.0 \%$, then a second measurement is made.

Upon observation of the second measurement, the average $m_{2}$ if formed. Because the second measurement is, loosely speaking, no better and no worse than the first one, the decision is based on the average. At the second step, if the average $m_{2}$ of the first two measurements is $<12.6 \%$, the decision "confirm LEU" is reached; if $\mathrm{m}_{2}$ exceeds $20.5 \%$, the decision "unable to confirm" is reached. If $m_{2}$ falls between $12.6 \%$ and $20.5 \%$, then a third measurement is made. 
The decision process continues, as indicated in Fig. 1 , at the $n^{\text {th }}$ step monitoring the average $m_{n}$ of the $n$ individual measurements observed to that point. If no decision has been reached after the $7^{\text {th }}$ measurement, then the $8^{\text {th }}$ and last measurement is mads. If $\pi_{8}$ exceeds $14.4 \%$, the decision "unable to confirm" is reached; otherwise, the decision "confirm LE'f" is reached. Because the values of $\left\{m_{n}\right\}$ should tend to converge to the actual enrichment, it is clear from Fig. I that when measurement conditions are good, decisions of high quality are reached quickly for actual enrichments in the anticipated range. Only when the observed measurements are "ambiguous" do decisions require a longer time.

Figure 2 depicts the modified SPRT, using the same parameter values as above, when the standard deviation of each measurement is $\sigma=108$. This standard deviation corresponds to poor--but under some circumstances, expected--measurement conditions. Because of the increased uncertainty, the "continue measuring" bands are wider than those in Fig. 1. More measurements are required, on the average, to reach decisions and those decisions are not of the same quality as those for the $\sigma=4 \%$ case.

From Figs. 1 and 2, a quick termination with the "unable to confirm" decision can be characterized. Reaching that decision relatively quickly can happen either as the result of an initial, very large measured value or as the result of several measured values whose average is large enough. Either wa.: sufficient doubt is cast on the credibility of Leu to preclud. confirmation with high confidence. Once a strong doubt has been created, it is not adequately removed by observing a small number of additional measured values internally consistent with those already available.

For example, Fig. l indicates that for the $o=4 \%$ case, an "unabie to confirm" decision would follow the fourth measurement if the average $\mathrm{m}_{4}$ of those measurements were equal to $18 \%$ (and if no decision har- been reached earlier). That the "unable to confirm" deciston came quickly did not mean that high conficience could be placed in an HEu claim; after all, $m_{4}=18 \%$ is slightly more supportive of LE' than HEU.

To quantify the performance of the modified SPRT, a simulation exercise was conducted. Taken as representative of potential measurement conditions based on GCEP-related activities are the following: 


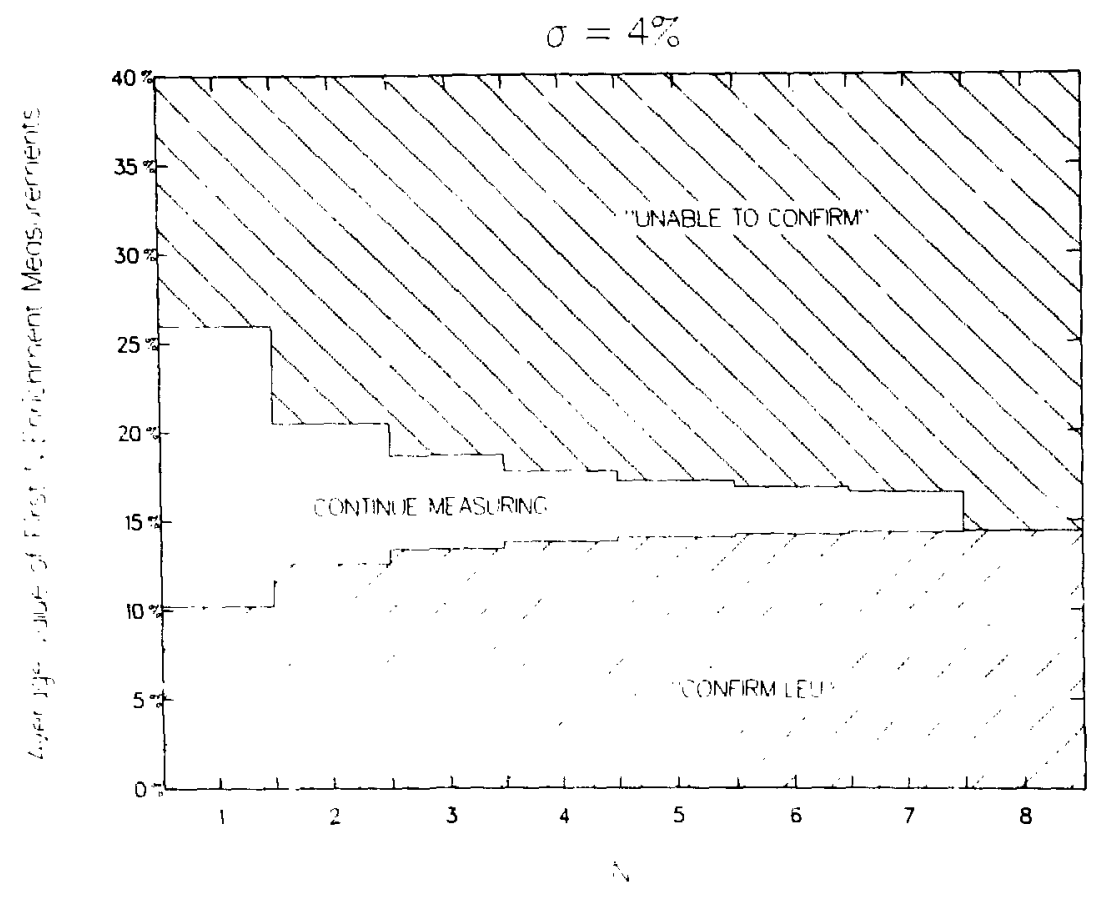

Fig. 1. Graphical representation of the suggested decision procedure when $\sigma=48$.

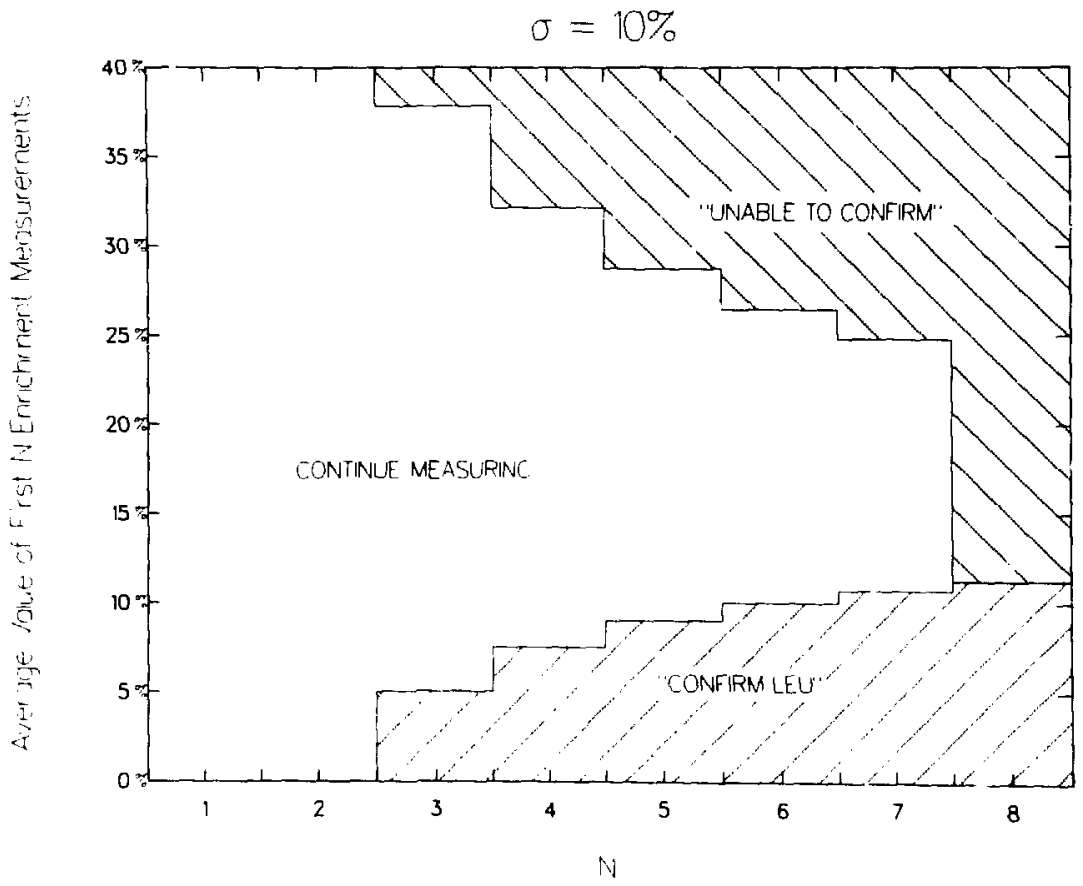

Fig. 2. Graphical representation of the suggested decision procedure when $\sigma=10 \%$. 
(a) a "clean pipe," where the standard deviation $\sigma$ of each individual measurement varies (linearly) from 48 for $3 \%$ actual enrichment to $6 \%$ for $20 \%$ actual enrichment; and

(b) a "dirty pipe," where the standard deviation o of each individual measurement varies (linearly) from $10 \%$ for $3 \%$ actual enrichment to 128 for $20 \%$ actual enrichment.

This situation is depicted in Fig. 3; note that the exiremes correspond to Figs. 1 and 2 when the enrichment is $3 \%$. (Asides: (1) In practice, standard deviations are not known exactly and are estimated based on counting statistics. It is important that count rates are such that the propagated standard deviations are very good; otherwise, the decision error rates are adversely affected. As mentioned in Sec. II, experimental work on this subject should be undertaken. (2) If measurement uncertainties were far better than indicated for the clean pipe, then different parameter values for $\theta_{0}$ and $\alpha$ in the modified SPRT should be used. At present, such a circumstance seems unlikely.)

Results from the simulation are presented in Table II. Under good measurement conditions, decisions are fast and accurate. For sub-58 enrichments, only one or two measurements are usually needed to make virtually error-free decisions. For $20 \%$ enrichment, four to seven measurements are usually needed and there is less than a 28 chance of erroneously concluding "confirm LEU." As always, the slowest decisions correspond to intermediate enrichments, but only when the enrichment is $14-18 \%$ are all eight measurements frequently (two-fifths of the time) needed.

At the other extreme of measurement conditions, performance of the modified SPRT is not nearly as good. For $3 \%$ enrichment, decisions require three to four measurements, on the average, and the decision error rate is $0.7 \%$. For $20 \%$ enrichment, eight measurements per decision are common. As would be expected, increased measurement uncertainty means that "confirm LEU" decisions cannot be made with high confidence as frequently as when uncertainties are smaller. Note from fig. 4 that the probability of an "unable to confirm" decision is uniformly higher over the LEU range for the dirty pipe than for the clean one. 


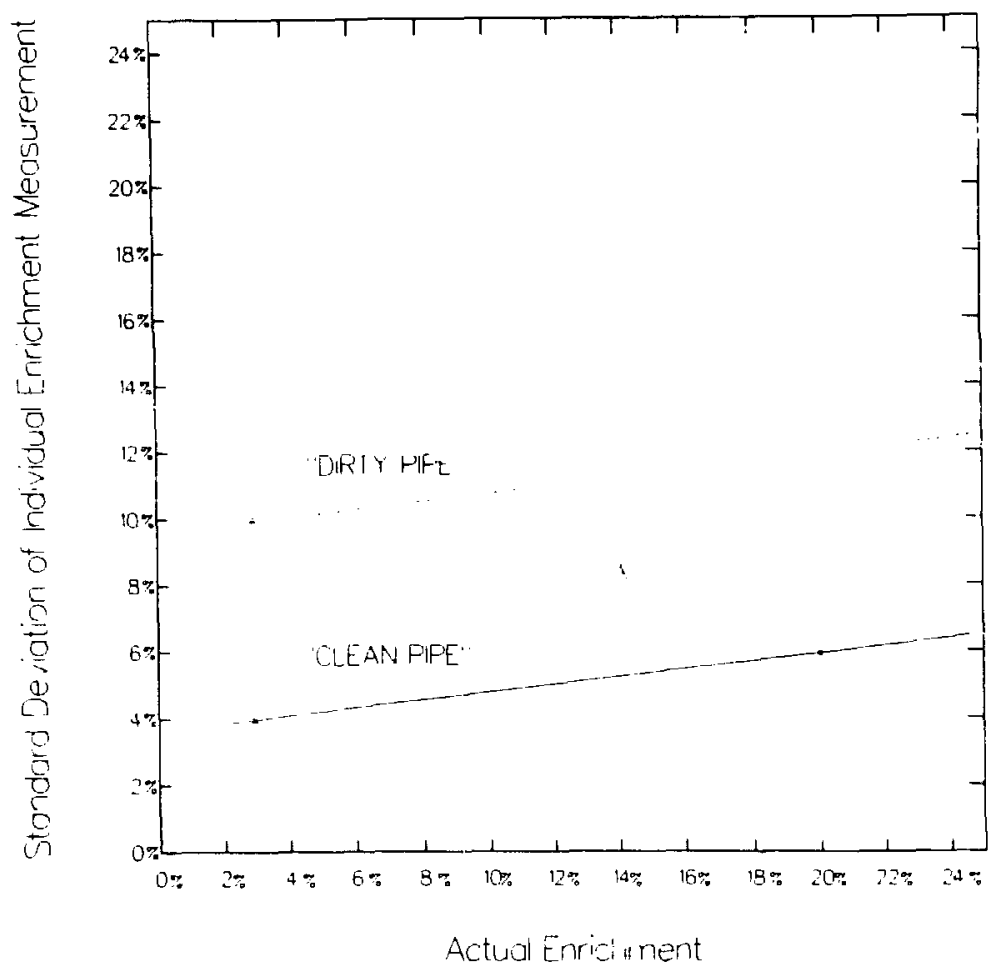

Fig. 3. Measurement standard deviations under anticipated conditions.

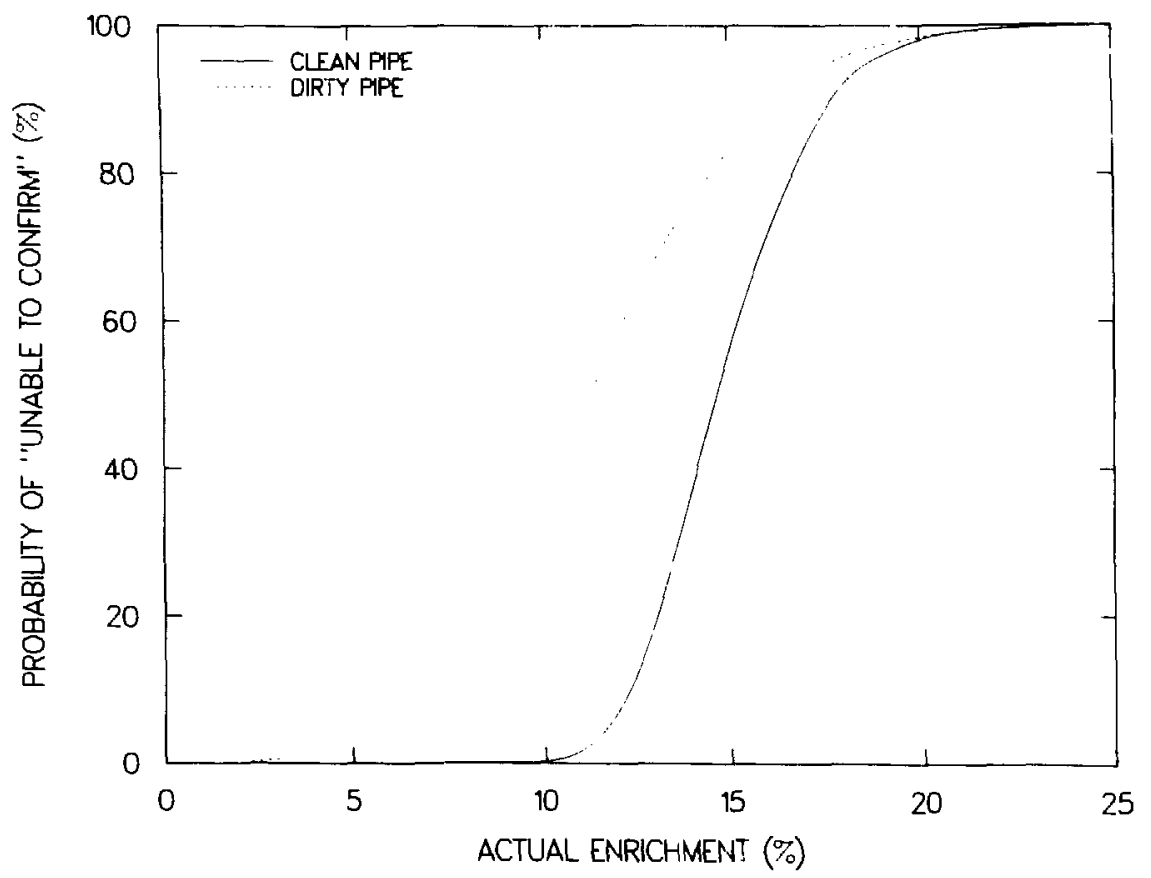

Fig. 4. Detection probability curve for the example of sec. $v$. 
TAB'.E II

PERFORMANCE* OF THE SUGGESTED MODIFIED SPRT PROCEDURE

Actual Enrichment

\begin{tabular}{|c|c|c|c|c|c|c|c|}
\hline "Clean Pipe" & 38 & 58 & 108 & 158 & 188 & 208 & $2 \underline{58}$ \\
\hline $\begin{array}{l}\text { Probability of } \\
\text { deciding "unable } \\
\text { to confirm" }\end{array}$ & 08 & 08 & 0.38 & 56.18 & 92.28 & $98.0 \%$ & $99.9 \%$ \\
\hline \multirow[t]{2}{*}{$\begin{array}{l}\text { Average number of } \\
\text { measurements required }\end{array}$} & 1.0 & 1.2 & 2.2 & 5.9 & 6.2 & 5.3 & 3.7 \\
\hline & \multicolumn{7}{|c|}{ Actual Enrichment } \\
\hline "Dirty Pipe" & 38 & -58 & 308 & 158 & 188 & $20 \%$ & 258 \\
\hline $\begin{array}{l}\text { Probah:1ity of } \\
\text { decid } 9 \text { "linable } \\
\text { to cor: } 2 . r m "\end{array}$ & 0.78 & 3.48 & 36.58 & 83.08 & 95.88 & 98.48 & 99.98 \\
\hline $\begin{array}{l}\text { Average number of } \\
\text { measurements required }\end{array}$ & 3.3 & 3.9 & 6.1 & 7.6 & 7.9 & 7.9 & 7.8 \\
\hline
\end{tabular}

VI. SUMMARY

As ravlewed in this report, the modified SPRT is an effective decisionmaking tool in the sequential measurement framework. The theoretical foundation for this tool has been well established in the statistical literature for many years. Simulation results applicable to the LFUA monitoring probiem indicate that fast, accurate decisions are reached under anticipated conditions. 
ACKNOWLEDGMENTS

The author thanks Rich strittmatter and John Pratt for their helpful comments during the preparation of this report.

REFERENCES

1. D. A. Close and J. C. Pratt, "The Measurement of Uranium Enrichment for Gaseous Uranium at Low Pressure," presented at the Seventh ESARDA Symposium on Safeguards and Nuclear Material Management, Liege, Belgium, riay 21-23, 1985 (to be published in Proceedings).

2. "Inspection Activities Associated with Limited-Frequency Unannounced Access Model Applied to Gas Centrifuge Type Enrichment Plants," Hexapartite Safeguards Project (February 1983).

3. R. R. Picard, "An Automated Decision Procedure for LFLA Monitorinf." Los Alamos National Jaboratory, Safeguards Systems Group draft report Q-4/85-351 (1985).

4. R. R. Plcard, "Application of Sequential Methods for Materials Balance Evaluation." Los Alamos National Laboratory. Safeguards Systems Group draft report Q-4/85-244 (May 1985).

5. A. Wald, Sejuential Analysis (Wiley and Sons, Inc., New York, 1947).

6. L. Weiss, "Testing One Simple Hypothesis Against Another," Annals of Mathematical statistics $24,273-281$ (1953).

7. L. LeCam, "Note on a Theorem of Lione 1 Weiss." Annals of Mathematical statistics $25,791-794$ (1954).

8. J. Kiefer and L. Weiss, "Some Properties of Generalized Sequentlal Probability Ratio Tests," Annals of Mathematical Statistics 28, 57-75 (1957).

9. T. W. Anderson, "A Modification of the Sequential Probability Ratio Test to Reduce the Sample Size," Annals of Mathemat1cal Statistics 31. 165-197 (1960).

10. G. Lorden, "2-SPRT's and the Modified Kiefer-Weiss Problem of Minimizing an Expected Sample Size," Annals of Statistics $\underline{4}, 281-291$ (1976). 